\title{
An Old Cold Killer...Overview of Perioperative Hypothermia
}

\author{
Rajkumar J.S ${ }^{1}$, Jayakrishna Reddy Aluru ${ }^{2}$, Shreya Rajkumar ${ }^{3}$, Rajkumar Anirudh ${ }^{4}$, \\ Dharmendra KR ${ }^{2}$, Syed Akbar ${ }^{2}$, Silas Danielraj J5* \\ ${ }^{I}$ FRCS. Lifeline Institute of Minimal Access \\ ${ }^{2}$ MS. Lifeline Institute of Minimal Access \\ ${ }^{3}$ MRCS, MS. Lifeline Institute of Minimal Access. \\ ${ }^{4}$ MRCS, DNB. Lifeline Institute of Minimal Access \\ ${ }^{5}$ MBBS, Dr Rela Institute and Medical Centre
}

*Corresponding Author: Silas Danielraj J, MBBS, Dr Rela Institute and Medical Centre

\begin{abstract}
Hypothermia occurring during surgery is said to occur in as high as $44.3 \%$ of all major intra operative procedures. It is defined as a fall of body temperature to 36-degree celsius or less. This is to report our experience about an episode of hypothermia in a patient who underwent extended abdominoplasty following bariatric surgery. Surgery was conducted under general anaesthesia with endotracheal intubation, and took 210 minutes overall, the surgical procedure being uneventful. However, during reversal prior to extubation the patient suddenly developed hypertonia and tonic movements resembling a seizure. She was not extubated; ventilation was continued and all the parameters were rechecked. Her blood pressure, heart rate, SpO2, ECG, creatinine and electrolytes were normal, but her core temperature was $34.5^{\circ} \mathrm{C}$, measured with an ear drum temp probe, signifying moderate hypothermia. Prompt warming technique with forced air was reinstituted and within 30 minutes she came back to normal with reversal of hypertonia. She made a complete recovery, with normal response to verbal commands. Thereafter she was extubated and had a smooth postoperative period with the exception of having a subcutaneous drainage tube left in situ for a longer period of time, asseroma is a frequent sequel to hypothermia. This report is presented, along with a comprehensive review of perioperative hypothermia and available methods of preventing its occurrence.
\end{abstract}

Keywords: Hypothermia, Intra-operative, Perioperative, Abdominoplasty.

Abbreviations: MGB-Mini Gastric Bypass, GA-General Anesthesia

\section{INTRODUCTION}

The frequency of hypothermia occurring during surgery (perioperative hypothermia) is said to be as high as $44.3 \%$ of all major intra-op procedures (range of 4-73\%) [1,2] .It is defined as a fall of body temperature to $36 \mathrm{C}$ or less [3].This is to report our experience about an episode of hypothermia in a patient who underwent extended abdominoplasty following bariatric surgery. This report is presented, along with a comprehensive review of perioperative hypothermia and available methods of preventing its occurrence

\section{CASE REPORT}

A 24-year old lady, with an initial body weight of $152 \mathrm{~kg}$ had presented for bariatric surgery. Following sleeve gastrectomy done in 2015, she had a $37 \mathrm{~kg}$ loss of weight, resulting in $115 \mathrm{~kg}$ final weight in 1 year. She sought more weight loss and a mini gastric bypass (MGB) surgery done in 2017, a powerful malabsorptive operation that brought down the body weight to $85 \mathrm{~kg}$. At this point of time in 2018 ten months post MGB, for sagging over the abdomen thighs and buttocks, she sought abdominoplasty (Figure 1) and body contouring.

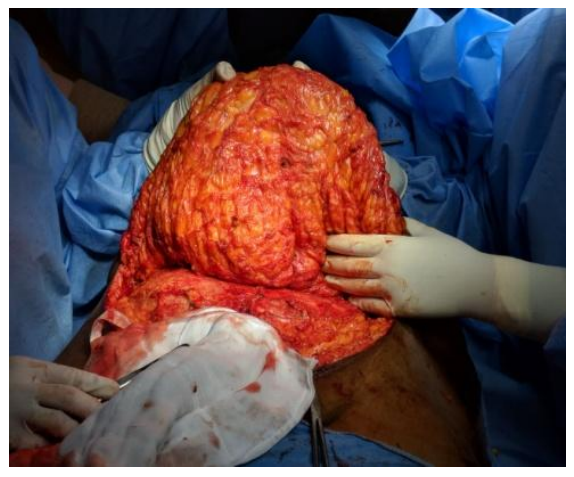

Figure1

Pre-operative investigations were all within normal limits. 
Under general anaesthesia (GA),with endotracheal intubation, the patient underwent abdominoplasty. The area from costal margin downwards was kept bare up to mid-thigh. Upper buttocks and post-lumbar area was also laid bare to enable pulling the skin flap forward, that forms an integral part of the body contouring.

Through an extended Pfannenstiel incision from iliac crest to iliac crest the lower abdomen was elevated, preserving the umbilical flap all the way to the costal margins. Flaps were extended laterally in a semi ellipse to include the lumbar area and the upper gluteal and thigh for tightening of the subplenus tissue of the region. After excision of the large lower abdomen ellipse and three smaller ellipses on either side in relation to lumbar area, the upper thighs and buttocks, the skin flaps were put together and sewn back primarily. The wound was closed in layers with drains in place. The blood loss was $300 \mathrm{ml}$, with no intraoperative blood transfusion, and no adverse events on vitals were seen intraoperatively.

During reversal from GA she went into sudden hypertonic posture and tonic seizure like contractions. She was not adequately responsive to verbal commands and pain stimuli;hence she was not extubated, ventilation continued and vitals monitored.

Serum levels of urea, creatinine, electrolytes, and Haemoglobin, Arterial Blood Gas and blood sugar estimations were within normal limits. Blood pressure and oxygen saturation were normal. Tachycardia was present $(115 / \mathrm{min})$. ECG could not be interpreted due to artefacts. Core temperature of 34.5 degree Celsius was measured with temperature probe measuring infrared heat generated by the ear drum (Braun ThermoScan). A diagnosis of perioperative hypothermia was made. She was wrapped up and a Bair Hugger warm forced air convector blanket was applied. Within 30 minutes her temperature returned to normal and she was wide awake, oriented and responsive. She was extubated soon after and made an uneventful recovery. In the post-operative period, there were no adverse events. There was no flap necrosis, fever or delayed infection. Drainage tube was left in situ, as hypothermia is associated with a seromacollection, and she was discharged on the 5thpost operative day.

\section{DISCUSSION}

Core temperature of less than 36-degree celsius is defined as hypothermia. A range of $35-35.9^{\circ} \mathrm{C}$ is mild, $34-34.9^{\circ} \mathrm{C}$ is moderate and below 34 is ${ }^{\circ} \mathrm{C}$ severe hypothermia $[1,2,3,4]$. Hypothermia during anaesthesia is not uncommon and can occur inadvertently $[2,5]$, of the heat transfer mechanism from surgical patients to environment, radiation is the major source of heat loss. Conductive loss is minimal. Convective loss of heat is proportional to square root of air speed which forms the basis of the wind chill factor, an aspect to be considered in an Operation Theatre (OT) with laminar air flow. Evaporation from surgical wounds contribute substantially to total heat loss. General anaesthesia impairs normal autonomic thermoregulatory control. Thermoregulatory system maintains core temperature within a few 10 ths of a ${ }^{\circ} \mathrm{C}$ of normal, which is approximately $37^{\circ} \mathrm{C}$. Anaesthesia induces inhibition of thermoregulation and combined with cold operating room conditions makes unwarmed patients hypothermic.

Under GA hypothermia causes an initial rapid temperature reduction, and a subsequent slower and a linear reduction in core temperature ultimately stabilizing and remains unchanged. Volatile anaesthetics cause vasodilatation and inhibit tonic vasoconstriction resulting in arteriovenous shunt dilatation. Anaesthetics also reduce metabolic rate by $20-30 \%$. Yet this reduction in heat production (metabolism) and increased heat loss is not enough to explain the $0.5-1.5^{\circ} \mathrm{C}$ decrease in core temperature during the first hour of anaesthesia. Anaesthesia induces vasodilatation and heat transfer from core to periphery causing warming of extremities at the expense of core temperature [6].After the initial distribution hypothermia, core temperature reduces in a slow linear way for 2 to 3 hours because heat loss exceeds heat production. After 3 to 4hours core temperature reaches a plateau for the duration of the surgery. In patients kept warm the core plateau is due to heat production equalling heat loss. This is not a steady state as body heat content continues to decrease despite core temperature remaining steady. [7, 8]

Normal Thermoregulation is usually done via positive and negative feedback and the mechanism essentially involves three phases, namely peripheral sensing, central regulation, and efferent response. Cold afferents are conducted via $\mathrm{A} \delta$ fibres and pain and warmth via C nerve fibres, with some overlap [9]. Centrally, hypothalamus integrates the inputs from skin, neuraxis, and deep tissues with 
threshold temperatures for each thermoregulatory response and these tissues give approximately $20 \%$ of thermal output to the central regulatory system.

In response to hypothermia, the autonomic effector responses are mostly by cutaneous vasoconstriction which counters heat loss. The digital blood flow via the $\mathrm{A}-\mathrm{V}$ shunts can vary from trivial to maximal levels with temperature changes restricted to within a few tenths of a degree $\mathrm{C}$. This is regulated by $\alpha$ adrenergic sympathetic nerves $[10,11]$.

Even a mild hypothermia of approximately 1 to $2{ }^{\circ} \mathrm{C}$ causes multiple issues. It prolongs postoperative anaesthesia recovery and duration of hospitalization, triples incidence of surgical wound infection [12] and hampers coagulation, increases blood loss and need for allogenic transfusion by $20 \%$.It also triples the chances of morbid myocardial outcomes ${ }^{13}$ and interferes with drug metabolism by increases the plasma concentration of propofol and muscle relaxants. The action of vecuronium is prolonged and the MAC of inhalational agents is decreased[14].In the post-op period hypothermia is physiologically stressful, increasing Blood pressure, Heart Rate and plasma catecholamines concentration. However, hypothermia has shown to improve outcomes of out of hospital cardiac arrest [15]. A beneficial effect is also seen in asphyxiated neonates [16]. Mild hypothermia of $34^{\circ} \mathrm{C}$ provides potential protection in neurosurgery [17]. Unlike in adults, non-shivering thermogenesis doubles heat production in infants. Sustained shivering gives $50-100 \%$ of metabolic heat in adults. Rapid tremor less than $250 \mathrm{~Hz}$ is unsynchronised muscle activity and probably is not central in origin. Along with this is a slow component 4 to 8 cycles/min, synchronous, waxing-waning pattern which is centrally mediated [18, 19].

\section{Patterns of Post-op Tremor:}

Postoperative shivering-tremor, the aetiology of which is unclear, has a $40 \%$ incidence. This increases oxygen consumption by $100 \%$. Most important factors are young age and core temperature. There are two distinct patterns:

A.Tonic pattern, which is more common, resembling normal shivering having a waxingwaning of 4 to 8 cycles/min [7].

B.Clonic $5-7 \mathrm{~Hz}$ bursting pattern looking like pathognomic clonus. Pathological spinal cord responses including clonus, nystagmus, and exaggerated deep tendon reflexes are common during general anaesthesia recovery [20].

Post anaesthetic shivering can be treated with skin surface warming as the regulatory system can withstand core hypothermia when cutaneous warming is increased. However cutaneous warming compensates only for small amounts of core temperature hypothermia and is not effective for core temperatures below $35^{\circ} \mathrm{C}$.

Other drugs to treat post anaesthetic shivering include areclonidine, tramadol, physostigmine, magnesium sulphate and dexmedetomidine. The mechanism of action is not known. Clonidine and dexmedetomidine reduce the vasoconstriction and shivering threshold which suggests that they are probably acting on central thermoregulatory system. Pethidine (meperidine) is more effective than equivalent analgesic doses of other mu agonists [21].

Preventing cutaneous heat loss due to ambient operating room conditions and to radiation and convection and evaporation from surgical site is done with the help of surgical drapes, cotton blankets and plastic sheets. The commonly used warming method is forced air which is more effective than circulating water mattresses and is cost effective [22]. Monitoring core body temperature during general anaesthesia is important to detect inadvertent hypothermia. Core temperature variation ranges from 0.5 to $1.5^{\circ} \mathrm{C}$ in first 30 minutes and is difficult to interpret. However, it is more important in cases where surgery lasts more than 1 hour, especially in cases under regional anaesthesia where changes in body temperature are anticipated. The temperature needs to be maintained above $36^{\circ} \mathrm{C}$. Electronic thermometers which are thermistors/thermocouples are accurate, disposable and inexpensive. Infrared monitors that deduce tympanic membrane temperature from outer ear are an alternative, though some authorities question their reliability. [23]

Most recommendations of various anaesthesia safety bodies towards temperature monitoring and maintenance strategies are that core body temperature is to be monitored for surgeries of 30 minutes duration or more and Efforts to maintain core temperature at $36^{\circ} \mathrm{C}$ [24].

\section{CONClusion}

Intra-op patient warming assumes great significance in conditions like prolonged surgery and large open wounds. In view of the adverse impact of hypothermia on the anaesthetised patient, its attendant co- 
morbidities, surgery and post-surgical complications, the importance of intra-operative core temperature monitoring to prevent hypothermia cannot be overemphasized.

\section{REFERENCES}

[1] Forkin KT, Nemergut EC. Miller's Anesthesia, 8th Edition. Anesthesiology. 2016 Apr 1

[2] Leslie K, Sessler DI. Perioperative hypothermia in the high-risk surgical patient. Best Pract Res Clin Anaesthesiol. 2003 Dec

[3] Kalra P. Miller's Anesthesia, Volumes 1 and 2, 7th Edition. Anesthesiology. 2010 Jan 1

[4] Bernard SA, Gray TW, Buist MD, Jones BM, Silvester W, Gutteridge G, et al. Treatment of Comatose Survivors of Out-of-Hospital Cardiac Arrest with Induced Hypothermia. $\mathrm{N}$ Engl $\mathrm{J}$ Med. 2002 Feb 21

[5] Amoateng-Adjepong Y, Del Mundo J, Manthous CA. Accuracy of an infrared tympanic thermometer. Chest. 1999 Apr

[6] Sessler DI. Perianesthetic thermoregulation and heat balance in humans. FASEB J. 1993 May

[7] Sessler DI. Perioperative thermoregulation and heat balance. Lancet. 2016 Jun 25

[8] Sessler DI. Temperature Monitoring and Perioperative Thermoregulation. Anesthesiology. 2008 Aug

[9] Denu ZA, Hailu YimerTawuye PS, Tawuye HY, Kassa AA. Perioperative Hypothermia and Predictors of Intra-Operative Hypothermia among Patients Operated at Gondar university Hospital from March to April 2015. J Anesth Clin Res. 2015 Aug 23

[10] Janicki PK, Higgins MS, Janssen J, Johnson RF, Beattie C. Comparison of two different temperature maintenance strategies during open abdominal surgery: upper body forced-air warming versus whole body water garment. Anesthesiology. 2001 Oct

[11] Kurz A, Sessler DI, Christensen R, Clough D, Plattner O, Xiong J. Thermoregulatory vasoconstriction and perianesthetic heat transfer. Acta AnaesthesiolScand Suppl. 1996

[12] Radauceanu DS, Dragnea D, Craig J. NICE guidelines for inadvertent peri-operative hypothermia. Anaesthesia. 2009 Dec.
[13] Rodrigues G, Prabhu R, Ravi C. Fast-track surgery: A new concept of perioperative management of surgical patients. J Heal Spec. 2013

[14] Reynolds L, Associate R, Beckmann J, Kurz A. Perioperative complications of hypothermia. Best Pract Res Clin Anaesthesiol. 2008

[15] Anderson JL, Morrow DA. Acute Myocardial Infarction. Campion EW, editor. N Engl J Med. 2017 May 25

[16] Maoulainine FMR, Elbaz M, Elfaiq S, Boufrioua G, Elalouani FZ, Barkane M, et al. Therapeutic Hypothermia in Asphyxiated Neonates: Experience from Neonatal Intensive Care Unit of University Hospital of Marrakech. Int J Pediatr [Internet].2017:1-7.

[17] Meloni BP, Campbell K, Zhu H, Knuckey NW. In search of clinical neuroprotection after brain ischemia: the case for mild hypothermia (35 degrees C) and magnesium. Stroke. 2009 Jun 1

[18] Delavar M, Akbarianrad Z, Mansouri M, Yahyapour M. Neonatal hypothermia and associated risk factors at baby friendly hospital in babol, iran. Ann Med Health Sci Res. 2014 Jul

[19] Mullany LC. Neonatal hypothermia in lowresource settings. Semin Perinatol. 2010 Dec

[20] Zimmerman B, Hubbard JB. Anatomy, Deep Tendon Reflexes (Stretch Reflexes).

[21] SCCM | Pharmacological Management of Therapeutic Hypothermia-Induced Shivering.

[22] Park FD, Park S, Chi S-I, Kim HJ, Seo K-S, Kim H-J, et al. Clinical considerations in the use of forced-air warming blankets during orthognathic surgery to avoid postanesthetic shivering. J Dent Anesth pain Med. 2015 Dec

[23] Aksoy F, Yıldırım YS, Veyseller B, Demirhan H. Aural and Nasal Temperature Measurements Using an Infrared Thermometer vs Conventional Axillary and Oral Measurements. Open Otorhinolaryngol J. 2014 Aug 7

[24] Bindu B, Bindra A, Rath G. Temperature management under general anesthesia: Compulsion or option. J Anaesthesiol Clin Pharmacol. 2018.

Citation: Rajkumar J.S, Silas Danielraj J et.al. An Old Cold Killer...Overview of Perioperative Hypothermia. ARC Journal of Anesthesiology. 2020; 5(2): 09-12. DOI: doi.org/10.20431/ $2455-9792$. 0502001 .

Copyright: (C) 2020 Authors. This is an open-access article distributed under the terms of the Creative Commons Attribution License, which permits unrestricted use, distribution, and reproduction in any medium, provided the original author and source are credited. 